Results: The committee's recommendations relate to the initial treatment of pediatric trauma, including stabilization and transfer phase and the first "golden hour." Recommendations for treatment in hospitals according to their levels include: National centers (Level 1 Trauma center), regional centers (Level 2 Trauma center), recommendations for trauma staffing (personnel), recommendations for upgrading equipment and infrastructure, training programs and refreshing knowledge and skills, and the development a PATLS course (Pediatric Advanced Trauma Life Support).

Conclusion: Writing a summary report, which was accepted by the Ministry of Health and by the National Committee for Trauma and Emergency Medicine, as a national work program for improving and upgrading child care trauma cases.

Prehosp Disaster Med 2017;32(Suppl. 1):s103-s104

doi:10.1017/S1049023X17002655

\section{Operating Room Management During Mass Casualties: A}

\section{New Checklist}

Joseph Mcisaac

Anesthesiology, University of Connecticut/Hartford Hospital, Hartford/CT/United States of America

Study/Objective: The American Society of Anesthesiology has released a new checklist for management of the operating theater during mass-casualty events.

Background: No prior system existed for operative management. Methods: Expert opinion from prominent trauma and disaster anesthesiologists.

Results: Refer to facility's Operations Manual; open up the appropriate annex.

Activate a call-in tree; assign an individual to activate, and use clerical personnel or automatic paging system, if available.

Assess status of Operating Rooms (ORs); determine staffing of OR's 0-2, 2-12, and 12-24 hours. Hold elective cases.

Alert current ORs; finish current surgical procedures as soon as possible, and prepare to receive trauma.

Assign staff; set up for trauma and emergency cases.

Anesthesia Coordinator should become OR Medical Director; work with OR Nursing Manager to facilitate communication, and coordination of staff and facilities.

Report OR status to the Hospital Command Center (HCC); enter telephone, email address of HCC.

Ensure adequate supplies; coordinate with anesthesia technicians' supply personnel, to ensure adequate supplies of fluids, medications, disposables, and other items.

Contact PACU; accelerate the transfer of patients to floors or ICU's, in preparation for a high volume of cases.

Anesthesiologist should act as liaison in Emergency Department (ED); send an experienced practitioner to the ED, to act as a liaison (your eyes \& ears), and keep communications open to the Anesthesia Coordinator.

Consider assembly of Stat Teams; use the combination of anesthesia, surgical, nursing, respiratory personnel to triage, as needed.

HAZMET/WMD event; review special personal protective procedures, such as DECON, and isolation techniques.
Consider if part of the OR, or hallways, should be considered "hot," or should have ventilation altered. Good resources include the CHEMM/REMM websites.

Coordinate with blood bank; verify blood availability.

Coordinate with other patient care areas: ICU's, OB, Peds, etc., to ensure continuity of care for new and existing patients.

Conclusion: This guidance provides a structured, task-based approach.

Prebosp Disaster Med 2017;32(Suppl. 1):s104

doi:10.1017/S1049023X17002667

Are Surgical Skills Under-Emphasized in Literature on Medical Response to Disasters? A Brief Review and Critical Analysis of the Literature with Emphasis on Low Resourced Populations

Paul J. Moroz, Steven R. Wilding', Nicholas W. Moroz ${ }^{3}$

1. Department Of Orthopaedic Surgery, University of Hawaii, Honolulu/HI/United States of America

2. Department Of Orthopaedic Surgery, Tripler Army Medical Centre, Honolulu/HI/United States of America

3. University of Guelph, Guelph/ON/Canada

Study/Objective: The literature on the medical response to disasters appears to underemphasize the importance of surgical skills, especially in Low- and Middle-Income Countries (LMIC).

Background: While emphasizing the important issues of medical aid, control of infections, or water and food security it is also recognized that the acute phase of disaster requires immediate or almost immediate surgery, for life and limb threatening surgical pathology. It is our hypothesis that the literature on surgery in first medical response to disasters is sparse, especially in vulnerable, low-resourced populations.

Methods: A PubMed advanced search using a standard Boolean search strategy was employed: "(disaster OR disaster response) AND (medical aid OR medical response OR humanitarian aid) AND (surgery OR surgical skills OR surgical procedure) AND (developing country OR austere environment OR low resource environment OR third world)". Subsets of this search strategy generated articles for review. Other search engines were examined using a similar search strategy.

Results: A Pubmed search strategy including "(disaster OR disaster response) AND (medical aid OR medical response OR humanitarian aid)" yielded 4,470 articles. If "(surgery OR surgical skills OR surgical procedure)" was added to this same search strategy then 519 articles (11.6\% of total) are identified. If "(developing country OR austere environment OR low resource environment OR third world)" are then added, then 21 articles $(0.5 \%$ of total) are identified. Of these 21 articles identified only $4(0.1 \%$ of total $)$ address the needs or issues of local surgical assets in LMIC, the remainder instead emphasizing surgical capacity of foreign medical teams in LMIC.

Conclusion: Our hypothesis is confirmed with only $11.6 \%$ of total articles on the medical response to disasters discussing surgical issues to any degree, despite significant surgical trauma seen in disasters, especially earthquakes. Only $0.5 \%$ of total 
articles discuss disaster-related surgical care in developing countries, with few discussing improving local country surgical assets. Most papers instead discuss the impact of foreign surgical teams, which are also clearly needed. Reasons for these overall findings are discussed. Opportunities to investigate the significant gap in surgical preparedness to disasters should be addressed. Research on improving local surgical assets in LMIC, in particular to address the acute phase of disasters, may be beneficial.

Prehosp Disaster Med 2017;32(Suppl. 1):s104-s105

doi:10.1017/S1049023X17002679

\section{Assessment of Advanced Life Support Training in Emergency Medical Personnel : a National Report of Thailand Chanisa Eksombatchai, Khrongwong Musikatavorn, Suthaporn Lumlertgul \\ Emergency Medicine, King Chulalongkorn Memorial hospital, and faculty of medicine, Chulalongkorn university, Bangkok/Thailand}

Study/Objective: To survey the number of Emergency Medical Service (EMS) personnel certified in Advanced Life Support (ALS) in Thailand.

Background: In Thailand, Emergency Medicine specialty has been established for only 17 years. In-depth knowledge of emergency medicine training in ALS and knowledge gap between each kind of personnel had never been studied.

Methods: Research was done in the form of survey research using a questionnaire. The surveys were allocated by postal mail to emergency rooms in 13 health districts throughout Thailand. The target group was emergency medical personnel who practiced in EMS of Thailand: Emergency Physicians (EP), General Physicians (GP), Paramedics, Emergency Nurse Practitioners (ENP), and Registered Nurses (RN). A selfadministered questionnaire consisting of personal and professional profiles, certificated status in ALS, and 50-questions of procedural competency assessment was used.

Results: According to 1,155 respondents (80\% response rate), the percentages of personnel who passed each of the ALS courses is as follows: Advanced Cardiac Life Support (ACLS) 81.56\%; Advanced Trauma Life Support (ATLS) 62.77\%; Pediatric ALS (PALS) 45.45\%; Prehospital Trauma Life Support (PHTLS) 15.84\%; Advanced Disaster Life Support (ADLS) 8.23\%; WINFOCUS Ultrasound ALS (WINFOCUS) 6.15\%; Advanced Hazmat Life Support (AHLS) 4.85\%; and others $1.9 \%$, respectively. There were $11.60 \%$ that had never been trained in ALS (Figure 1). The average competency score was $34.02 / 50(\mathrm{SD}=8.81)$. $\mathrm{EP}$ had a significantly higher mean competency score than GP $(\mathrm{P}<.01)$. ENP had a significantly higher mean score compared with $\mathrm{RN}(\mathrm{P}<.01)$; there was no significant difference between ENP and Paramedics $(\mathrm{P}=.06)$. Other factors that significantly associated with competency score were gender, hospital type, and work experience. Most of the participants (92\%) desired additional training in ALS.

Conclusion: Emergency medical personnel in Thailand should be supported to train in ALS in order to comprehend standard emergency medical practice throughout the country.

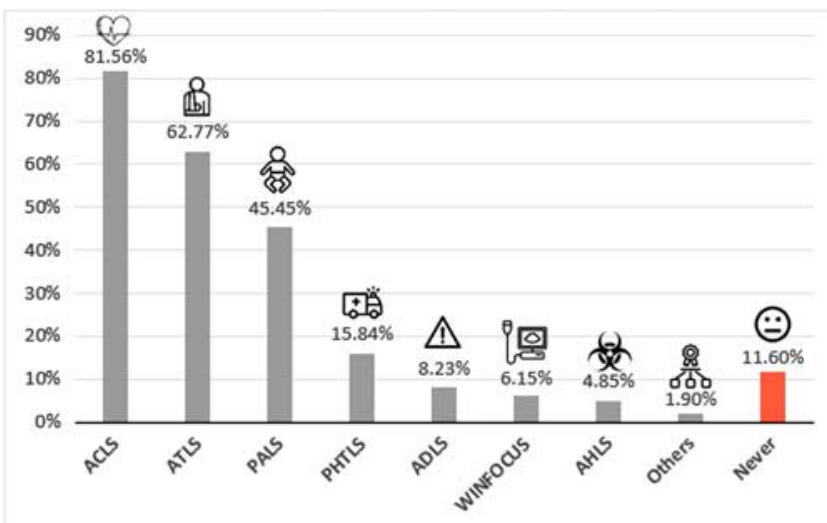

Figure 1. Percentages of emergency medical personnel who passed each of advanced life support courses. ACLS, ATLS, PALS, PHTLS, ADLS, WINFOCUS, AHLS, HAZMAT life support, Never passed any advanced life support courses

Prehosp Disaster Med 2017;32(Suppl. 1):s105

doi:10.1017/S1049023X17002680

\section{Senior Nursing Students Working as an American}

Red Cross Volunteer: Virtual Case Workers

Montray Smith ${ }^{1}$, Nancy Kern ${ }^{2}$

1. School Of Nursing, University of Louisville, Louisville/KY/United States of America

2. Disaster Health Services, American Red Cross, Louisville/KY/ United States of America

Study/Objective: The American Red Cross (ARC) Kentucky Region, and the University of Louisville (UL) School of Nursing, have facilitated a student clinical experience in disaster preparedness. Fall 2016, a pilot project developed from the Red Cross calls for virtual volunteers to assist with disaster relief services to natural disasters. In response, UL- $4^{\text {th }}$ semester Community Practicum Leadership Practicum students were trained as volunteer virtual caseworkers for the ARC Disaster Services Disaster Action Team.

Background: The American Red Cross is mandated to provide assistance to victims of single- and multi-family fires, in addition to other disasters. Nurses assist caseworkers to provide support to clients, to assist with immediate response, and recovery needs by addressing health needs. Involvement of pre-licensure nursing students as caseworkers allows them to utilize their community leadership education to facilitate clients' recovery.

Methods: The $4^{\text {th }}$ semester Community Health baccalaureate nursing students were trained and responded as American Red Cross virtual caseworkers; as part of a disaster response action team, they served individuals and families affected by home fires in the Kentucky and Southern Indiana region. The group received required training through online modules and in-person orientation to prepare for the role.

Results: The results showed that the nursing students and American Red Cross staff required additional education. The current on-boarding processes needed improvement, and case 